\title{
RESPONSE TO EVOLVE BIOSYSTEMS' COMMENTS
}

\author{
George Kent
}

I appreciate having representatives of Evolve Biosystems, David Kyle and Tracy Shafizadeh, comment on my recent critical article in World Nutrition, available at

https://worldnutritionjournal.org/index.php/wn/article/view/597/533 I would like to clarify my views.

First, I did not say a product cannot be both patentable and safe. My concern is that something produced in a new way should not be assumed to be safe. They say the GRAS Panel assessed the safety through review of a broad range of scientific studies and assessed it as safe. How can their report be accessed?

Second, my article reproduced an image from the Evolve Biosystems website with the caption, "9 Out of 10 babies are missing B. infantis." Presumably referring to their Evivo product, another image had the caption " 9 out of 10 babies need it now". Does this this refer to 9 out of 10 babies in the world, in the U.S., or somewhere else?

Where did they get this information? Drs. Kyle and Shafizadeh said, "the absence of B. infantis in infants from most Western populations today is exemplified by many publications in the peer reviewed literature where the infant gut microbiome has been investigated.” The fact that there have been many studies shows that are some cases, but they do not show that that it is widespread. The three articles they cited (Bäckhed, Frese, and Vatenen) did not provide a basis for a 9-out-of-10 estimate anywhere.

In their response to my article they said., " $B$. infantis has been disappearing in the Western world for the past 50 years or more.” They did not explain the source of this information, or the evidence regarding the causes and consequences of this disappearance.

Third, it is true they did not say there is a widespread deficiency in breastmilk. However, the 9out-of-ten caption along with the offer of a fortifier for breastmilk might lead some mothers to think this means their breastmilk is somehow inadequate. Perhaps Evolve Biosystems could adjust their promotional material to prevent such misinterpretations.

Fourth, if the reduction or absence of B. Infantis is a major problem, why haven't we heard more about it from major health agencies at the national and global levels?

Fifth, in my World Nutrition article, I asked, "What argument did they use to get \$40 million in funding for promoting the product in Asia, to help infants suffering from severe acute malnutrition (SAM) through the restoration of the gut microbiome.” They did not address that question. I hope this helps to clarify my concerns.

Aloha, George Kent 